\title{
Fatores relacionados ao óbito pela Influenza Pandêmica A (H1N1) 2009 em pacientes tratados com Oseltamivir
}

\author{
Factors related to death by the Pandemic Influenza A (H1N1) 2009 in patients treated with Oseltamivir
}

Factores relacionados con la muerte por la Influenza Pandémica A (H1N1) 2009

en pacientes tratados con Oseltamivir

\section{Luana Lenzi', Lineu Roberto da Silva", Ângela Maron de Mello"'", Mônica Holtz Cavichiolo Grochocki ${ }^{\mathrm{Iv}}$, Roberto Pontarolov}

\author{
'Universidade Federal do Paraná, Setor de Ciências da Saúde, Laboratório de Química Medicinal Farmacêutica, \\ Programa de Pós-Graduação. Curitiba-PR, Brasil. \\ "Secretaria de Estado da Saúde do Paraná, Centro de Produção e Pesquisa de Imunobiológicos, \\ Seção de Biologia Medica Ambiental. Piraquara-PR, Brasil. \\ I'I Fundação Nacional de Saúde, Coordenação Regional do Paraná, Divisão de Vigilância Epidemiológica. Curitiba-PR, Brasil. \\ IV Secretaria de Estado da Saúde do Paraná, Consórcio Paraná Saúde. Curitiba-PR, Brasil. \\ v Universidade Federal do Paraná, Setor de Ciências da Saúde, Departamento de Farmácia. Curitiba-PR, Brasil.
}

Submissão: 07-04-2012 Aprovação: 23-07-2013

\section{RESUMO}

Este estudo pretende identificar os fatores relacionados ao óbito na Influenza Pandêmica A(H1N1) 2009 em pacientes tratados com Oseltamivir. Trata-se de um estudo observacional e retrospectivo, realizado com dados de pacientes que apresentaram diagnóstico laboratorial da doença. Os dados foram coletados dos formulários de notificação da doença, pertencentes a uma base de dados do Ministério da Saúde. As análises estatísticas foram realizadas pelos testes de qui-quadrado, t de Student e por regressão logística, considerando significativos os valores de $p<0,05$. A maior ocorrência de óbitos foi observada nos pacientes com idade entre 20 e 59 anos, de baixa escolaridade, com a presença de comorbidades, não vacinados, tratados tardiamente e que apresentavam sintomas mais severos da infecção. A identificação de fatores de risco para o óbito reforça a necessidade de prevenção e assistência precoce, principalmente na presença de fatores que aumentam a gravidade clínica da doença.

Descritores: Vírus da Influenza A Subtipo H1N1; Fatores de Risco; Causas de Morte; Prevenção \& Controle; Letalidade.

\section{ABSTRACT}

This study aimed to identify factors related to death in Pandemic Influenza $A(H 1 N 1) 2009$ in patients treated with Oseltamivir. It is an observational and retrospective study, carried out with data of patients who had presented laboratorial diagnosis of the illness. Data were collected from the notification forms of the disease, belonging to a database of the Ministry of Health. Statistical analysis was performed by chi-square, Student t test and logistic regression, considering significant $p$ values $<0,05$. The highest mortality was observed in patients aged between 20 and 59 years, low schooling, with the presence of comorbidities, not vaccinated, treated late and had more severe symptoms of infection. The identification of risk factors for death reinforces the need for prevention and early care, especially in the presence of factors that increase the clinical severity of disease.

Key words: Influenza A Virus, H1N1 Subtype; Risk Factors; Cause of Death; Prevention and Control; Lethality.

\section{RESUMEN}

Este estudio se propone identificar los factores relacionados con la muerte dela influenza pandémica A(H1N1)2009 en pacientes tratados con Oseltamivir. Es un estudio observacional y retrospectivo, realizado con datos de los pacientes que presentan diagnóstico de laboratorio. Los datos fueron recogidos de los formularios de notificación de la enfermedad, pertenecientes a una base de datos del Ministerio de Salud. Los análisis estadísticos se realizó mediante las pruebas del qui-cuadrado, t de Student y regresión logística, en vista de significativo los valores de $p<0,05$. La mayor mortalidad se observó en pacientes con edades entre 20 y 59 años, baja escolaridad, con la presencia de comorbilidades, no vacunados, tratados tardíamente y que habían síntomas más graves de la infección. La identificación de factores de riesgo para la muerte refuerza la necesidad de la prevención y atención temprana, especialmente en la presencia de factores que aumentan la gravedad de la enfermedad.

Palabras clave: Subtipo H1N1 del Virus de la Influenza A; Factores de Riesgo; Causas de Muerte; Prevención \& Control; Letalidad.

\section{AUTOR CORRESPONDENTEＬuana LenziＥ-mail: luanalnz@yahoo.com.br}




\section{INTRODUÇÃO}

Durante o ano de 2009, foram inúmeros os casos confirmados de infecção pelo vírus da Influenza A (H1N1) pandêmico. Segundo dados da Organização Mundial da Saúde, 208 países, territórios e comunidades reportaram casos confirmados laboratorialmente da doença, incluindo 12.799 óbitos ${ }^{(1)}$.

As áreas mais atingidas foram a Europa, África do Norte e o Sudeste da Ásia. No Brasil, as regiões Sul e Sudeste foram as mais afetadas. Na região Sul, a taxa de incidência de Síndrome Respiratória Aguda Grave (SRAG) por Influenza Pandêmica A (H1N1) 2009 foi de 66,2 casos para cada 100 mil habitantes ${ }^{(2)}$.

Para o tratamento da infecção, análises iniciais demonstraram que esta nova cepa de Influenza A (H1N1) era sensível aos inibidores da neuraminidase. Dentre os medicamentos dessa classe terapêutica está o Oseltamivir ${ }^{(3-7)}$.

No Brasil, segundo o Protocolo de Manejo Clínico e Vigilância Epidemiológica da Influenza na sua versão I, de 08 de julho de 2009, o tratamento com Oseltamivir foi indicado a todos os indivíduos que apresentaram Síndrome Respiratória Aguda Grave (SRAG) e fator de risco para agravamento, de acordo com a avaliação médica. Além disso, o antiviral estava indicado para pacientes de grupos específicos como os portadores de comorbidades e crianças com quadro clínico sugestivo de síndrome gripal, independente da gravidade, da faixa etária e da presença ou não de fatores de risco. Nos casos suspeitos, que preenchiam a definição de caso de SRAG, o tratamento com o antiviral fosfato de Oseltamivir deveria ser iniciado o mais breve possível e dentro das primeiras 48 horas após o início dos sintomas, sendo mantido por um período de 5 dias $^{(8)}$.

Segundo informações do detentor do registro do medicamento, a exposição ao Tamiflu (Oseltamivir) foi estimada em pelo menos 21,1 milhões de pacientes durante o período pandêmico de primeiro de maio a 31 de dezembro de 2009. Segundo informe publicado pelo Ministério da Saúde, quase 900.000 tratamentos com Oseltamivir foram distribuídos para todas as unidades federativas do Brasil, até a semana epidemiológica $39^{(9)}$.

Observa-se uma sobrevida estatisticamente significante em pacientes diagnosticados com a Influenza A (H1N1) tratados com Oseltamivir. Os pacientes tratados apresentaram reduções significativas na magnitude e na duração da replicação viral, redução da severidade e duração dos sintomas, redução de complicações e de hospitalizações de adultos, bem como a diminuição de infecções respiratórias agudas e do período de transmissibilidade ${ }^{(10-12)}$. Entretanto, estudos têm reportado cerca de $15 \%$ de óbitos entre os pacientes tratados com Oseltamivir ${ }^{(13)}$.

Este estudo pretende identificar quais os fatores relacionados aos indivíduos e à doença, que mesmo na utilização do tratamento, apresentaram relação direta com o óbito, podendo sugerir redução da efetividade do antiviral, ou maior gravidade na infecção.

\section{MÉTODOS}

Trata-se de um estudo observacional e retrospectivo, utilizando como fonte de informação o registro de casos de Influenza Pandêmica A(H1N1) 2009 do Sistema de Informação oficial, Sistema Nacional de Informação de Agravos de Notificação (Sinan) do Ministério da Saúde (formulários de notificação). Os dados foram coletados na Secretaria de Estado da Saúde do Paraná.

A população do estudo foi composta por pacientes que apresentaram a infecção pelo vírus da Influenza Pandêmica A (H1N1) 2009, com diagnóstico realizado por RT-PCR (Reverse transcription of polymerase chain reaction) e que foram tratados com Oseltamivir.

Para o estudo dos fatores que apresentaram relação com o óbito foram incluídas as seguintes variáveis: idade, presença de fator de risco conhecido para agravamento da influenza destacados na ficha de investigação do Sinan (sim ou não, separadamente), os principais sinais e sintomas, internação, e as datas do início dos sintomas, do início do tratamento com Oseltamivir e do óbito (quando falecidas).

$\mathrm{Na}$ análise estatística buscou-se comparar as taxas de cura e de óbito nos pacientes tratados com Oseltamivir, categorizados de acordo com a presença ou ausência de cada uma das variáveis pesquisadas. Essas comparações foram realizadas pelos teste de qui-quadrado (variáveis nominais) e teste t de Student (variáveis contínuas). A pesquisa dos fatores de risco para o óbito foi realizada por regressão logística, utilizando-se como medida de associação a Odds Ratio (OR), com Intervalo de Confiança de 95\% (IC95\%). A pesquisa da proporção de distribuição das variáveis em cada grupo foi realizada pelo teste-z. As análises estatísticas foram realizadas com o software SPSS 17.0, considerando como significativos os valores de $p<0,05$.

Este projeto cumpre os princípios contidos na Declaração de Helsinki, atende a legislação específica do Brasil e foi aprovado pelo Comitê de Ética em Pesquisa institucional, sob o número de registro 938.063.10.06 e CAAE: 0038.0.091.000-10.

\section{RESULTADOS}

Foram utilizados nesse estudo, dados de 1.827 pacientes, os quais foram diagnosticados laboratorialmente e apresentavam informações declaradas acerca do uso do Oseltamivir na Ficha Individual de Notificação. Dentre esses pacientes, verificou-se que, 201 (11\%) evoluíram ao desfecho óbito.

Sabendo-se que a presença de alguns fatores pode aumentar a predisposição ao óbito pela Influenza, foram avaliadas as taxas de cura e óbito nos pacientes que receberam o medicamento Oseltamivir, segundo características demográficas e clínicas. Dessa forma, objetivou-se verificar quais as variáveis que apresentaram relação significativa nos desfechos da doença, mesmo na vigência do uso do fármaco. Os resultados encontrados estão representados nas tabelas 1 e 2 .

Conforme observado na tabela 1 , a idade média dos pacientes que usaram o fármaco e morreram, foi maior do que a idade média dos pacientes tratados que evoluíram para a cura. O gênero não apresentou significância na relação com os desfechos. Os percentuais de óbito e cura foram estatisticamente semelhantes nos grupos divididos segundo essa característica.

Em relação ao nível de escolaridade, os menores percentuais de óbito ocorreram nos pacientes com mais de 12 anos de estudo e em crianças fora da idade escolar. O resultado do teste- $z$ confirmou que nesses grupos as proporções de cura foram significativamente maiores que as de óbito. 
Tabela 1 - Taxas de óbito e cura, segundo características dos pacientes tratados com Oseltamivir $(\mathrm{N}=1.827)$.

\begin{tabular}{|c|c|c|c|c|c|c|}
\hline \multicolumn{2}{|c|}{ Características } & Total & $\begin{array}{c}\text { Óbito } \\
n=201\end{array}$ & $\begin{array}{c}\text { Cura } \\
\mathrm{n}=\mathbf{1 . 6 2 6}\end{array}$ & $\begin{array}{c}\text { Análise } \\
\text { estatística }\end{array}$ & Teste-z \\
\hline \multicolumn{2}{|l|}{ Idade } & $23,3( \pm 23,4)$ & $37,6( \pm 15,6)$ & $21,6( \pm 15,6)$ & $p=0,000$ & -- \\
\hline \multirow{4}{*}{ Gênero } & Masculino & $797(43,6 \%)$ & $86(10,8 \%)$ & $711(89,2 \%)$ & \multirow{4}{*}{$p=0,064$} & \multirow{2}{*}{--} \\
\hline & Feminino & $1030(56,4 \%)$ & $115(11,2 \%)$ & $915(88,8 \%)$ & & \\
\hline & 0 a 4 anos & $256(14 \%)$ & $28(10,9 \%)$ & $228(89,1 \%)$ & & -- \\
\hline & 5 a 11 anos & $627(34,3 \%)$ & $56(8,9 \%)$ & $571(91,1 \%)$ & & -- \\
\hline \multirow[t]{3}{*}{ Escolaridade } & $>12$ anos & $187(10,2 \%)$ & $5(2,7 \%)$ & $182(97,3 \%)$ & \multirow{3}{*}{$p=0,000$} & $\mathrm{C}>\mathrm{O}$ \\
\hline & Não informado & $485(26,5 \%)$ & $103(21,3 \%)$ & $382(78,7 \%)$ & & -- \\
\hline & Não se aplica* & $272(14,9 \%)$ & $9(3,3 \%)$ & $263(96,7 \%)$ & & $\mathrm{C}>\mathrm{O}$ \\
\hline \multirow{2}{*}{ Hospitalização } & Sim & $943(51,6 \%)$ & $195(20,7 \%)$ & $748(79,3 \%)$ & \multirow{2}{*}{$p=0,000$} & $\mathrm{O}>\mathrm{C}$ \\
\hline & Não & $884(48,4 \%)$ & $6(0,7 \%)$ & $878(99,3 \%)$ & & $\mathrm{C}>\mathrm{O}$ \\
\hline $\begin{array}{l}\text { Tempo para iniciar } \\
\text { tratamento (dias) }\end{array}$ & $\begin{array}{l}\text { Mínimo: } 0 \\
\text { Máximo: } 49\end{array}$ & $2,5( \pm 2,7)$ & $5,4( \pm 5,1)$ & $2,1( \pm 2,0)$ & $p=0,000$ & -- \\
\hline
\end{tabular}

NOTA: $\mathrm{C}=$ Cura; $\mathrm{O}=$ Óbito; ${ }^{*}$ Não se aplica = crianças fora da idade escolar

Tabela 2 - Taxas de óbito e cura, segundo as comorbidades, nos pacientes tratados com Oseltamivir ( $\mathrm{N}=1.827)$.

\begin{tabular}{|c|c|c|c|c|c|c|}
\hline \multicolumn{2}{|l|}{ Características } & Total & $\begin{array}{c}\text { Óbito } \\
n=273\end{array}$ & $\begin{array}{c}\text { Cura } \\
\mathrm{n}=\mathbf{4 . 4 6 7}\end{array}$ & \multirow[t]{2}{*}{$\begin{array}{l}\text { Análise } \\
\text { estatística }\end{array}$} & Teste-z \\
\hline \multirow{2}{*}{ Presença de Comorbidade } & Não & $1.357(74,3 \%)$ & $123(9,1 \%)$ & $1.234(90,9 \%)$ & & $\mathrm{C}>\mathrm{O}$ \\
\hline & Sim & $470(25,7 \%)$ & $78(16,6 \%)$ & $392(83,4 \%)$ & $p=0,000$ & $\mathrm{O}>\mathrm{C}$ \\
\hline Número de comorbidades & $\begin{array}{l}\text { Mínimo: } 0 \\
\text { Máximo: } 7\end{array}$ & $0,38( \pm 0,77)$ & $0,88( \pm 1,2)$ & $0,35( \pm 0,72)$ & $p=0,000$ & -- \\
\hline \multirow{2}{*}{ Cardiopatias } & Não & $1.755(96,1 \%)$ & $180(10,2 \%)$ & $1.575(89,8 \%)$ & \multirow{2}{*}{$p=0,000$} & $\mathrm{C}>\mathrm{O}$ \\
\hline & Sim & $72(3,9 \%)$ & $21(29,2 \%)$ & $51(70,8 \%)$ & & $\mathrm{O}>\mathrm{C}$ \\
\hline \multirow{2}{*}{ Pneumopatias } & Não & $1.641(89,8 \%)$ & $182(11,1 \%)$ & $1.459(88,9 \%)$ & \multirow{2}{*}{$p=0,718$} & \multirow{2}{*}{--} \\
\hline & Sim & $186(10,2 \%)$ & $19(10,2 \%)$ & $167(89,8 \%)$ & & \\
\hline \multirow{2}{*}{ Nefropatias } & Não & $1.803(98,7 \%)$ & $194(10,8 \%)$ & $1.609(89,2 \%)$ & \multirow{2}{*}{$p=0,004$} & $\mathrm{C}>\mathrm{O}$ \\
\hline & Sim & $24(1,3 \%)$ & $7(29,2 \%)$ & $17(70,8 \%)$ & & $\mathrm{O}>\mathrm{C}$ \\
\hline \multirow{2}{*}{ Imunodepressão } & Não & $1.772(97 \%)$ & $186(10,5 \%)$ & $1.586(89,5 \%)$ & \multirow{2}{*}{$p=0,000$} & $\mathrm{C}>\mathrm{O}$ \\
\hline & Sim & $55(3 \%)$ & $15(27,3 \%)$ & $40(72,7 \%)$ & & $\mathrm{O}>\mathrm{C}$ \\
\hline \multirow{2}{*}{ Tabagismo } & Não & $1.715(93,9 \%)$ & $177(10,3 \%)$ & $1.538(89,7 \%)$ & \multirow{2}{*}{$p=0,000$} & $\mathrm{C}>\mathrm{O}$ \\
\hline & Sim & $112(6,1 \%)$ & $24(21,4 \%)$ & $88(78,6 \%)$ & & $\mathrm{O}>\mathrm{C}$ \\
\hline \multirow{2}{*}{ Diabetes } & Não & $1.767(96,7 \%)$ & $180(10,2 \%)$ & $1.587(89,8 \%)$ & \multirow{2}{*}{$p=0,000$} & $\mathrm{C}>\mathrm{O}$ \\
\hline & Sim & $60(3,3 \%)$ & $21(35 \%)$ & $39(65 \%)$ & & $\mathrm{O}>\mathrm{C}$ \\
\hline \multirow{2}{*}{$\begin{array}{l}\text { Hipertensão Arterial } \\
\text { Sistêmica (HAS) }\end{array}$} & Não & $1.762(96,4 \%)$ & $184(10,4 \%)$ & $1.578(89,6 \%)$ & \multirow{2}{*}{$p=0,000$} & $\mathrm{C}>\mathrm{O}$ \\
\hline & Sim & $65(3,6 \%)$ & $17(26,2 \%)$ & $48(73,8 \%)$ & & $\mathrm{O}>\mathrm{C}$ \\
\hline \multirow{2}{*}{ Obesidade } & Não & $1806(98,8 \%)$ & $195(10,8 \%)$ & $1611(89,2 \%)$ & \multirow{2}{*}{$p=0,010$} & $\mathrm{C}>\mathrm{O}$ \\
\hline & Sim & $21(1,2 \%)$ & $6(28,6 \%)$ & $15(71,4 \%)$ & & $\mathrm{O}>\mathrm{C}$ \\
\hline
\end{tabular}

NOTA: $C=$ Cura; $\mathrm{O}=$ Óbito 
Quanto à hospitalização, observamos que o maior percentual de óbitos ocorreu no grupo de pacientes hospitalizados. Os casos graves apresentaram rápida evolução, e apesar das intervenções clínicas recebidas durante o internamento, foram verificados casos de óbito entre os pacientes internados. Em relação ao tempo para iniciar o tratamento após o início dos sintomas, o intervalo foi significativamente maior nos pacientes que evoluíram para o óbito.

Os dados da tabela 2 indicam que a taxa de óbito foi significativamente maior nos pacientes que apresentavam outras comorbidades associadas à infecção pela Influenza Pandêmica $\mathrm{A}(\mathrm{H} 1 \mathrm{~N} 1) 2009$, sendo que estes pacientes apresentaram maior proporção de óbitos do que de cura, conforme resultado do teste-z. Em relação ao número de comorbidades presentes e associadas à infecção, observamos que o valor médio é significativamente superior nos pacientes que evoluíram ao desfecho óbito. Quando as comorbidades são analisadas individualmente, observamos que somente a pneumopatia não apresentou significância na relação com os desfechos.

Tabela 3 - Regressão logística univariada para identificação dos fatores de risco dos paicentes tratados com Oseltamivir.

\begin{tabular}{|c|c|c|c|}
\hline & $\mathbf{N}$ & OR & IC95\% \\
\hline Idade & $23,4( \pm 16,4)$ & 1,05 & $1,04-1,06$ \\
\hline Com algum grau de escolaridade & 1.056 & 0,5 & $0,4-0,6$ \\
\hline Sem escolaridade* & 771 & 2,0 & $1,5-2,5$ \\
\hline Hospitalização & 943 & 38,1 & $16,8-86,4$ \\
\hline Tempo para iniciar o tratamento ${ }^{\dagger}$ (dias) & $2,5( \pm 2,7)$ & 1,4 & $1,3-1,5$ \\
\hline Com comorbidade & 470 & 2,0 & $1,5-2,7$ \\
\hline Sem comorbidade & 1.357 & 0,5 & $0,4-0,7$ \\
\hline $\mathrm{N}^{0}$ Comorbidades associadas & Mín:0 / Máx:7 & 1,6 & $1,4-1,8$ \\
\hline Cardiopatia & 72 & 3,6 & $2,1-6,1$ \\
\hline Nefropatia & 24 & 3,4 & $1,4-8,3$ \\
\hline Imunodepressão & 55 & 3,2 & $1,7-5,9$ \\
\hline Tabagismo & 112 & 2,4 & $1,5-3,8$ \\
\hline Diabetes & 60 & 6,2 & $3,6-10,8$ \\
\hline HAS & 65 & 3,0 & $1,7-5,4$ \\
\hline Obesidade & 21 & 3,3 & $1,3-8,6$ \\
\hline Dispnéia & 985 & 9,2 & $5,8-14,8$ \\
\hline Hemoptise & 8 & 14,7 & $5,3-40,7$ \\
\hline Pneumonia & 8 & 13,8 & $3,3-58,2$ \\
\hline Não vacinados contra Influenza & 1514 & 3,5 & $1,4-8,8$ \\
\hline Vacinados contra Influenza & 152 & 0,3 & $0,1-0,7$ \\
\hline
\end{tabular}

NOTA; *Sem escolaridade = crianças fora da idade escolar, analfabetos ou escolaridade não informada. ${ }^{\dagger} A$ partir do início dos sintomas.
Já as comorbidades cardiopatias, nefropatias, imunodepressão, tabagismo, diabetes e obesidade, influenciaram significativamente nos desfechos dos pacientes tratados, aumentando as taxas de óbitos dos portadores dessas comorbidades mesmo na vigência do uso do antiviral Oseltamivir.

Quanto aos sinais e sintomas da infecção, 89,6\% dos pacientes tratados que foram a óbito apresentaram febre, 94\% tosse, $90 \%$ dispneia e $62,7 \%$ mialgia. Oito pacientes apresentaram pneumonia e oito apresentaram hemoptise. Em ambos os grupos, $5(62,5 \%)$ pacientes foram a óbito. Outro sintoma que apresentou relação com o aumento da taxa de óbito foi a dispneia - um dos principais sintomas na definição de caso de SRAG, sendo que $18,4 \%$ dos pacientes que apresentaram este sintoma foram a óbito.

Em relação à vacinação, $152(8,3 \%)$ pacientes haviam sido vacinados contra a Influenza. É importante salientar que a vacina não é disponibilizada para todos os grupos, sendo elegíveis para receberem, as crianças com idade entre 6 meses e 2 anos, as gestantes, os portadores de comorbidades, os idosos e os profissionais da saúde. Dentre os pacientes vacinados, 3,3\% evolui ao desfecho óbito. Já no grupo de pacientes não vacinados, a taxa de letalidade foi de 10,8\%. A diferença entre as taxas de letalidade em relação á vacinação, foi significativa $(p=0,004)$, indicando que a proporção de cura foi maior no grupo que havia recebido a vacina contra a Influenza, enquanto que o óbito foi superior no grupo que não havia sido vacinado.

A tabela 3 contém os resultados da análise univariada por regressão logística com as variáveis que apresentaram relação significativa com as taxas de cura e óbito. Esta análise buscou avaliar qual o impacto desses fatores na razão de chances para a ocorrência do desfecho óbito.

Com relação à idade, observou-se que a cada ano de vida, aumenta-se em 5,5\% a razão de chances para o óbito. Este resultado indica que a idade é um fator de risco para o óbito. A figura 1 revela que a maior ocorrência de óbitos foi observada nos pacientes com idade entre 20 e 59 anos.

Em relação à escolaridade, verificou-se que pacientes sem nenhum nível de escolaridade (analfabetos ou fora da idade escolar) apresentaram aumento de 2 vezes na razão de chances para o óbito. A figura 2 ilustra o percentual de óbitos para os diferentes níveis de escolaridade, evidenciando que os percentuais são decrescentes, conforme aumenta o nível de escolaridade do indivíduo.

O tempo médio para início do tratamento, após o inicio dos sintomas, foi de 2,5 dias. Conforme resultado da regressão logística, a cada dia de intervalo para início do tratamento, aumenta-se em $40 \%$ a razão de chances para o óbito.

Pacientes com comorbidade apresentaram 2 vezes mais chances de óbito do que os pacientes sadios (sem nenhuma comorbidade associada). Em relação ao número de comorbidades 
associadas, cada comorbidade somada, aumenta em $60 \%$ a razão de chances para o óbito. Em relação às comorbidades isoladas, a que mais incrementou a razão de chances para o óbito foi o diabetes. A ausência de comorbidades apresentou-se como fator de proteção ao óbito.

Figura 1 - Divisão dos óbitos nos pacientes tratados com Oseltamivir por faixa etária.

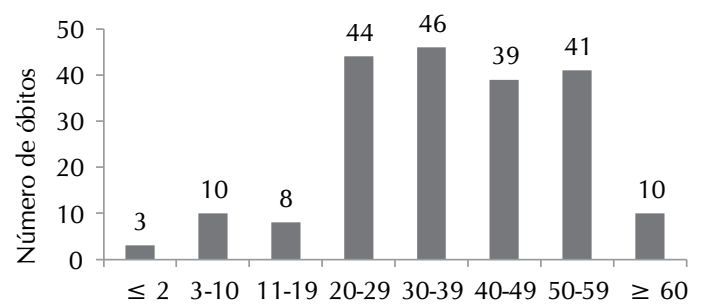

Faixa Etária (anos)

Figura 2 - Percentual de óbitos nos pacientes tratados com Oseltamivir conforme o nível de escolaridade.

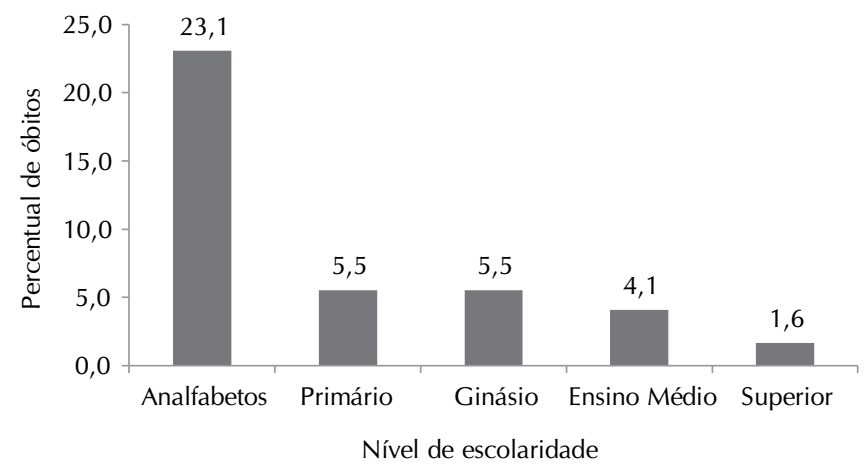

NOTA: Primário $=1^{a}$ a $4^{a}$ série do Ensino Fundamental; Ginásio $=5^{a} \mathrm{a}$ $8^{a}$ série do Ensino Fundamental.

Os pacientes não vacinados apresentaram maior razão de chances para o óbito, comparados aos pacientes vacinados. Além disso, a vacinação mostrou-se como um fator de proteção ao óbito pela doença, ao reduzir $70 \%$ a razão de chances para o óbito.

\section{DISCUSSÃO}

A idade já foi associada como fator de risco por outros estudos. Na Califórnia, observou-se que a taxa de mortalidade foi maior nos pacientes com idade superior a 50 anos $^{(14)}$. Outros estudos revelaram que na Alemanha, pacientes com idade igual ou superior a 60 anos apresentaram as maiores taxas de mortalidade $^{(15)}$, e a mediana da idade de pacientes internados em unidade de terapia intensiva na Espanha foi de 40 anos (0$90)^{(16)}$. Acredita-se que em parte, pacientes adultos podem ter sido poupados devido à imunidade pré-existente pelo contato prévio a cepas antigenicamente semelhantes a da pandemia.
Entretanto, uma vez infectados e hospitalizados, apresentaram elevadas proporções de óbitos, especialmente naqueles com mais de 60 anos de idade ${ }^{(14)}$. Indivíduos com idade superior a 30 anos apresentam maior prevalência de comorbidades, sugerindo maior acúmulo de fatores de risco para agravamento e óbito por influenza.

A maior taxa de óbito observada entre os pacientes hospitalizados não indica que a hospitalização tenha relação causal com o óbito, mas, sim, que os casos mais graves foram hospitalizados. Dessa forma, a hospitalização sugere um falso fator prognóstico para o óbito, pois os óbitos foram mais frequentes entre os pacientes hospitalizados porque eram estes os que apresentavam maior gravidade clínica, incluindo internamento em Unidade de Terapia Intensiva (UTI).

A ausência de relação entre a pneumopatia e o óbito por influenza nos pacientes desse estudo difere dos resultados publicados por outra pesquisa ${ }^{(15)}$, podendo ser explicada pela idade dos indivíduos pneumopatas. Uma análise do perfil dos pacientes que apresentavam pneumopatias, revelou que a maior parte eram crianças com idade entre 3 e 10 anos e, conforme discutido anteriormente, essa faixa etária apresentou menor risco de óbito. Esse dado pode justificar a exclusão da pneumopatia como fator de risco nessa população. Já a relação das demais comorbidades como fatores de risco para agravamento e óbito pela Influenza Pandêmica A(H1N1)2009 é confirmada pelos resultados de outros estudos ${ }^{(15-16)}$.

A relação entre o tempo para iniciar o tratamento a partir do início dos sintomas confirma a importância do tratamento precoce para a cura do paciente, conforme já relatado por outros estudos ${ }^{(15,17)}$. O tratamento com Oseltamivir em até dois dias após o início dos sintomas (tratamento precoce) foi associado à redução em $42 \%$ na probabilidade de ocorrência de uma ou mais infecções secundárias ${ }^{(18)}$.

Em relação aos sintomas, observou-se que a manifestação de dispnéia, hemoptise e pneumonia, aumentaram a razão de chances para o óbito e podem ser claramente associados à maior gravidade clínica da infecção.

A diminuição dos casos de óbito em relação à vacinação contra a Influenza e a revelação desta como fator de proteção ao óbito sugere impacto positivo do procedimento na redução da gravidade da doença. Além disso, a diminuição do número de óbitos nos pacientes com idade igual ou superior a 60 anos, conforme evidenciado pela figura 1 sugere aquisição de um efeito protetor conferido pela imunização a partir das vacinas, há anos administradas nesta camada da população, conforme discutido por outro autor ${ }^{(19)}$. Assim, esses pacientes, anteriormente reconhecidos como de maior risco para o óbito por influenza, possivelmente graças à imunidade cruzada, apresentaram neste evento pandêmico, menor número de óbitos.

A caracterização dos casos graves tem sido a chave para identificar os fatores de risco para complicações e óbitos por Influenza. Os resultados desse estudo que avaliam os fatores de risco nos pacientes tratados com o antiviral Oseltamivir, podem ser observados de duas maneiras distintas. Primeiro, os achados confirmam os dados já publicados na literatura em relação aos fatores de risco para agravamento e óbito pela 
infecção e ressalta a importância de outros ainda não mencionados, como a escolaridade, a vacinação e o número de comorbidades associadas. Segundo, sugere que apesar da eficácia do antiviral, ocorreram óbitos principalmente entre os pacientes que apresentavam fatores de risco e/ou foram tratados tardiamente. Essa última observação permite sugerir que a cura esperada com o tratamento pode ser comprometida pela presença dos fatores de risco ou pela dificuldade na assistência à saúde do paciente. Durante uma pandemia, o elevado número de casos e a velocidade com que ocorrem, sobrecarregam os serviços e geram escassez de recursos físicos e humanos, dificultando a assistência ao paciente. Dessa forma, o conhecimento acerca dos pontos estratégicos de cuidado ao paciente e em relação à intervenção a saúde são de fundamental importância no sucesso terapêutico.

\section{CONCLUSÕES}

Os fatores relacionados ao óbito pela Influenza Pandêmica $\mathrm{A}(\mathrm{H} 1 \mathrm{~N} 1) 2009$ nos pacientes tratados com Oseltamivir foram: a idade, a baixa escolaridade, o tempo para iniciar o tratamento, a presença de outras comorbidades, tais como as cardiopatias, nefropatias, imunodepressão, tabagismo, diabetes, hipertensão arterial e obesidade, o número de comorbidades associadas e a não vacinação contra a Influenza. Os pacientes apresentaram, significativamente, maiores taxas de óbito quando expostos a esses fatores, os quais aumentam significativamente as razões de chance para a ocorrência desse desfecho. Por fim, as evidências permitem concluir que o tratamento precoce com Oseltamivir ( $<48$ horas após inicio dos sintomas) reduziu os casos de óbito.

\section{REFERÊNCIAS}

1. World Health Organization [homepage na internet]. Pandemic (H1N1) 2009 - update 91 [acesso 14 jul 2010]. Disponível em: http://www.who.int/csr/don/2010_03_12/en/ index.html.

2. Portal da Saúde [homepage na internet]. Informe Epidemiológico. Situação epidemiológica da Influenza Pandêmica (H1N1) 2009 no mundo e no Brasil, até a Semana Epidemiológica 47 de 2009 [acesso em 20 jun 2010]. Disponível em: http://portal.saude.gov.br/portal/arquivos/ pdf/boletim_influenza_se_47.pdf.

3. Baz M, Abed $Y$, Nehmé B, Boivin G. Activity of the oral neuraminidase inhibitor A-322278 against the Oseltamivir-resistant $\mathrm{H} 274 \mathrm{Y}$ (A/H1N1) influenza virus mutant in mice. Antimicrob Agents Chemother 2009;53(2):791-3.

4. Chang LY, Shih SR, Shao PL, Huang DT, Huang LM. Novel swine-origin Influenza virus A (H1N1): the first pandemic of the 21st century. J Formos Med Assoc 2009;108(7):526-32.

5. Gerloff NA, Kremer JR, Mossong J, Opp M, Muller CP. Genomic diversity of Oseltamivir-resistant influenza virus $A(H 1 N 1)$, Luxembourg, 2007-08. Emerg Infect Dis 2009;15(9):1523-4.

6. Tanaka T, Nakajima K, Murashima A, Garcia-Bournissen F, Koren G, Ito S. Safety of neuraminidase inhibitors against novel Influenza A ( $11 \mathrm{~N} 1)$ in pregnant and breastfeeding women. CMAJ 2009;7;181(1-2):55-8.

7. Dutkowski R. Oseltamivir in seasonal Influenza: cumulative experience in low- and high-risk patients. J Antimicrob Chemother 2010;65(Suppl 2):ii11-ii24.

8. Porta da saúde [homepage na internet]. Protocolo de manejo clínico e vigilância epidemiológica da Influenza [acesso em 20 jun 2010]. Disponível em: http://portal. saude.gov.br/portal/arquivos/pdf/protocolo_de_manejo_ clinico_05_08_2009.pdf

9. Agência Nacional de Vigilância Sanitária [homepage na internet]. Informe SNVS/Anvisa/GFARM $\mathrm{n}^{\circ}$, de $1^{\circ}$ de abril de 2010. Oseltamivir no Brasil: uma análise das notificações de suspeitas de eventos adversos encaminhadas à Anvisa, maio a dezembro de 2009 [acesso em 23 jan 2011]. Disponível em: http://portal.anvisa.gov.br/wps/ wcm/connect/9f345080474580fc8d0fdd3fbc4c6735/ Oseltamivir.pdf?MOD = AJPERES

10. Hanshaoworakul W, Simmerman JM, Narueponjirakul U, Sanasuttipun W, Shinde V, Kaewchana S, et al. Severe Human Influenza Infections in Thailand: Oseltamivir Treatment and risk factors for fatal outcome. PLos ONE 2009;4(6):e6051.

11. Treanor JJ, Hayden FG, Vrooman PS, Barbarash R, Bettis $\mathrm{R}$, Riff $\mathrm{D}$, et al. Efficacy and safety of the oral neuraminidase inhibitor Oseltamivir in treating acute Influenza: a randomized controlled trial. US Oral Neuraminidase Study Group. JAMA 2000;283(8):1016-24.

12. Huerta García A, González Sánchez N, Torres Martí A. Influenza A (H1N1): manifestaciones clínicas e indicaciones profilácticas y terapéuticas. Arch Bronconeumol 2010;46(Suppl. 2):19-23.

13. Cui W, Zhao H, Lu X, Wen Y, Zhou Y, Deng B, et al. Factors associated with death in hospitalized pneumonia patients with $2009 \mathrm{H} 1 \mathrm{~N} 1$ influenza in Shenyang, China. BMC Infect Dis 2010;10:145.

14. Louie JK, Jean C, Acosta M, Samuel MC, Matyas BT, Schechter R. A Review of adult mortality due to 2009 Pandemic (H1N1) Influenza a in California. PLoS One 2011;6(4):e18221.

15. Wilking $\mathrm{H}$, Buda $\mathrm{S}$, von der Lippe $\mathrm{E}$, Altmann D, Krause G, Eckmanns T, et al. Mortality of 2009 pandemic influenza $\mathrm{A}(\mathrm{H} 1 \mathrm{~N} 1)$ in Germany. Euro Surveill 2010;15(49). pii: 19741.

16. Santa-Olalla Peralta $P$, Cortes García $M$, Limia Sánchez A, Andrés Prado J, Pachón Del Amo I, Sierra Moros MJ, et al. [Critically ill patients with 2009 pandemic influenza A (H1N1) infection in Spain: factors associated with death, April 2009-January 2010]. Rev Esp Salud Pública 2010;84(5):547-67. Spanish. 
17. Mikić D, Nozić D, Kojić M, Popović S, Hristović D, Dimitrijević RR, et al. Clinical manifestations, therapy and outcome of pandemic influenza A (H1N1) 2009 in hospitalized patients. Vojnosanit Pregl 2011;68(3):248-56.

18. Goldstein E, Cowling BJ, O'Hagan JJ, Danon L, Fang VJ, Hagy A, et al. Oseltamivir for treatment and prevention of pandemic influenza $\mathrm{A} / \mathrm{H} 1 \mathrm{~N} 1$ virus infection in households, Milwaukee, 2009. BMC Infect Dis 2010;10:211.

19. Ferrer ALM, Marcon SS, Santana RG. Hospital morbidity among elderly patients, before and after Influenza vaccination in the state of Paraná. Rev Latino-Am Enferm 2008;16(5):832-7. 